SISSA $/ 4 / 06 / \mathrm{EP}$

ULB-TH/06-03

hep-th/0602015

\title{
Bubbling AdS and Vacuum String Field Theory
}

\author{
L. Bonora ${ }^{(a) 1}$, C.Maccaferri ${ }^{(b)}{ }^{2}$, R.J.Scherer Santos ${ }^{(a) 3}$, D.D.Tolla ${ }^{(a)} 4$ \\ (a) International School for Advanced Studies (SISSA/ISAS) \\ Via Beirut 2-4, 34014 Trieste, Italy, and INFN, Sezione di Trieste \\ (b) Physique Théorique et Mathématique, Université Libre de Bruxelles 8 International \\ Solvay Institutes, ULB Campus Plaine C.P. 231, B-1050 Bruxelles, Belgium
}

\begin{abstract}
We show that a family of $1 / 2-\mathrm{BPS}$ states of $\mathcal{N}=4 \mathrm{SYM}$ is in correspondence with a family of classical solutions of VSFT with a $B$-field playing the role of the inverse Planck constant. We show this correspondence by relating the Wigner distributions of the $N$ fermion systems representing such states, to low energy space profiles of systems of VSFT D-branes. In this context the Pauli exclusion principle appears as a consequence of the VSFT projector equation. The family of $1 / 2-\mathrm{BPS}$ states maps through coarse-graining to droplet LLM supergravity solutions. We discuss the possible meaning of the corresponding coarse graining in the VSFT side.
\end{abstract}

Keywords: 1/2-BPS states, String Field Theory, Superstars, Coarse-graining

\footnotetext{
${ }^{1}$ bonora@sissa.it

${ }^{2}$ carlo.maccaferri@ulb.ac.be

${ }^{3}$ scherer@sissa.it

${ }^{4}$ tolla@sissa.it
} 


\section{Introduction}

The essence of this paper is the observation that there is a remarkable correspondence between states one meets in the framework of the AdS/CFT duality, and solutions of vacuum string field theory (VSFT). The correspondence is simply sketched and is far from exhaustive (mainly because we do not know enough about supersymmetric VSFT), but it is very suggestive and, if confirmed, it could lead to very interesting consequences. Roughly speaking it goes as follows. In the framework of type IIB superstring theory, AdS/CFT duality establishes a correspondence between $\mathcal{N}=4$ superconformal $U(N)$ gauge field theory on the boundary of $A d S_{5}$ and supergravity on the background $A d S_{5} \times S^{5}$. In the strongest formulation the correspondence is between the two theories as a whole. Here we will limit our consideration to a class of $1 / 2-$ BPS states which can be formulated as composite of the $U(N)$ gauge theory scalars. In general they can be cast in the form of Schur polynomials, and thus they are in one to one correspondence with Young diagrams, represented (in the case of giant gravitons [5], for instance) by columns of maximal size (number of boxes) $N$. On the supergravity side they correspond to $1 / 2$ BPS states that are solutions to the supergravity equations of motion. The latter represent localized states in the AdS geometry that wrap around $S^{3}$ cycles of $S^{5}$; they are stabilized by their angular momentum $J$ in $S^{5}$, with the condition $J \leq N$. There are other significant 1/2-BPS states with mass $\sim N^{2}$, the superstars [8]. They correspond to Young diagrams represented by approximate triangles. On the supergravity side these are singular 1/2-BPS states with a naked singularity, which are regarded as solutions on the verge of developing a black-hole horizon due to the quantum corrections. Giant gravitons and superstars are two examples of a zoo of new entities that can be constructed in similar ways.

An interesting question is the following one: what is the precise relation between a state in gauge field theory and the corresponding supergravity solution? More precisely, how does the geometry that characterize the latter arise from the former (which, at first sight, is a totally ungeometrical object)? The answer seems to be coarse-graining: geometry arises from averaging details of the quantum states in the gauge theory side.

The argument brought forth by [1, 2, 3] goes as follows. One remarkable aspect of the above gauge field theory states is that they can be represented also in terms of $N$ fermionic oscillators in a harmonic potential. The correspondence can be once again established via Young diagrams: quantum systems with the same Young diagram describe the same quantum state. This lends itself to a very interesting development: to quantum systems of this type we can associate in a one-to-one way Wigner distribution functions. In general, to a point-like system in a $(q, p)$ phase space we can associate a Wigner distribution function $W(q, p)$. This is nothing but the bosonization of the original fermion system, but $W(q, p)$ is also very close to a probability distribution in phase space. In this way we can associate a Wigner distribution to any state, such as the vacuum, "black rings", superstars, etc. Now, it so happens that these Wigner distributions are characterized in the large $N$ limit by (coarse-grained) profiles that can be matched to the corresponding geometry (droplets) of the $1 / 2$ BPS supergravity solutions.

With the above premise, the point we want to make in this paper is that the Wigner distributions for the above introduced states naturally appear in VSFT. More precisely, the same profiles appear as low energy space profiles of VSFT solutions. 
Vacuum string field theory (VSFT) is a version of Witten's open string field theory which is conjectured to represent string theory at the tachyon condensation vacuum [29]. Its action is formally the same as the original Witten theory except that the BRST charge takes a simplified form: it has been argued that it can be expressed simply in terms of the ghost creation and annihilation operators. By virtue of this simplification it has been possible to determine exact classical solutions which have been shown to represent D-branes. The existence of such solutions confirms the conjecture at the basis of VSFT ${ }^{5}$. It is possible to introduce families of such solutions, spanned by Laguerre polynomials. Any sum of distinct solutions is also a solution. It is also possible to introduce a constant background B-field in the internal directions and obtain, in the low energy limit, a space profile for these kind of solutions. The space profiles obtained in this way for a large family of VSFT solutions are (remarkably) the same as the Wigner distributions of a corresponding family of half-BPS states introduced above.

This correspondence leads us to a related subject: open-closed string duality as seen from the SFT point of view. A.Sen has recently conjectured, 30, that open string theory might be able to describe all the closed string physics, at least in a background where $\mathrm{D}_{-}$ branes are present. In this sense VSFT should be a privileged vantage point: the tachyon condensation vacuum physics can only represent closed string theory and thus VSFT should be able to describe closed string theory in the sense of [30]. The existence of the D-brane solutions mentioned above is a confirmation of this. However these D-brane solutions are expressed as squeezed (or related) states. At most, in the presence of a background B field, we can produce a space profile thereof. However it has not been known so far how to associate a corresponding geometry. The correspondence between space profiles and Wigner distributions may be the clue: by interpreting a space profile as a Wigner distribution, we can reconstruct a half-BPS state and as a consequence arrive at some definite geometry, which is the coarse-grained averaging over the corresponding fermion systems.

The above is the motivation of our research. However it must be said immediately that we are still far from a complete understanding of all the aspects involved. The big missing block is supersymmetric vacuum string field theory and its solutions. Should we know them we would be able to confirm the above suggestions, which therefore, at this stage, remain mostly conjectural, although not unmotivated.

The paper is organized as follows. In the next section we give a brief introduction of $1 / 2-\mathrm{BPS}$ states in CFT. We insist in particular in their representation by means of fermion systems of harmonic oscillators. Then we introduce the corresponding $1 / 2-\mathrm{BPS}$ supergravity solutions. Finally we describe in some detail the Wigner distributions, which are conjectured to be, in the large $N$ limit, the bridge between them. In section 3 we recall what is relevant in this context about VSFT solutions. In section 4 we establish the correspondence between $N$ fermion systems and VSFT solutions and make a list of facts supporting it. Section 5 is devoted to a critical assessment of the conjectural aspects of our paper.

\footnotetext{
${ }^{5}$ Recently there has been a very important breakthrough in OSFT: an analytic solution representing the tachyon condensation vacuum has been determined by Martin Schnabl, 40]. This will certainly shed new light on tachyon condensation and will hopefully clarify the role and status (is it only an effective theory?) of VSFT in it.
} 


\section{Half-BPS solutions}

In the field theory side of the AdS/CFT correspondence, half-BPS multiplets of $\mathcal{N}=4$ Yang-Mills theory fall into representations $(0, l, 0)$ of the $S O(6)$ R-symmetry group. Highest weight states can be constructed as gauge invariant polynomials of a complex scalar field $X$. The conformal dimension of the latter is $\Delta=1$ and the $U(1) \mathrm{R}$-charge $J=1$, where $U(1) \in S O(6)$. A highest weight therefore satisfies $\Delta=J$. Basically such states are constructed out of multiple traces of $X$. The most general state of this type of charge $n$ takes the form

$$
\left(\operatorname{tr}\left(X^{l_{1}}\right)\right)^{k_{1}}\left(\operatorname{tr}\left(X^{l_{2}}\right)\right)^{k_{2}} \ldots\left(\operatorname{tr}\left(X^{l_{p}}\right)\right)^{k_{p}}
$$

where the integers $l_{i}, k_{i}$ form a partition of $n: \sum_{i=1}^{p} l_{i} k_{i}=n$. A basis for these states is given by the degree $n$ Schur polynomials of the group $U(N)$. These in turn correspond to Young tableaux of maximal column length $N$. Therefore we can classify these highest weight states (chiral primaries) by means of Young diagrams [9, 10, 11.

It can be shown that they can be represented in another useful way. In fact their correlators can be related, by using the canonical approach, by correlators of a suitable (time-dependent) matrix model with a quadratic potential 9, 18. The matrix model can be solved also in another way, by diagonalizing it and producing in this way a Vandermonde determinant Jacobian factor [11]. The latter can be lifted to the exponential giving rise to a repulsive potential among the eigenvalues. The result is that we can interpret the eigenvalues $l_{i}$ as a system of $N$ fermionic oscillators with Hamiltonian $H=\sum_{i} l_{i}^{\dagger} l_{i}+\frac{1}{2}$. The energy levels of this system are given by $E_{i}=n_{i}+\frac{1}{2}$, where $n_{i}$ are nonnegative integers. The corresponding wavefunctions are given in terms of Slater determinants

$$
\Psi\left(l_{1}, l_{2}, \ldots, l_{n}\right) \sim e^{-\sum_{i} \frac{\lambda_{i}{ }^{2}}{2}} \operatorname{Det}\left(\begin{array}{cccc}
H^{n_{1}}\left(l_{1}\right) & H^{n_{1}}\left(l_{2}\right) & \ldots & H^{n_{1}}\left(l_{N}\right) \\
H^{n_{2}}\left(l_{1}\right) & H^{n_{2}}\left(l_{2}\right) & \ldots & H^{n_{2}}\left(l_{N}\right) \\
: & : & : & : \\
: & : & : & : \\
H^{n_{N}}\left(l_{1}\right) & H^{n_{N}}\left(l_{2}\right) & \ldots & H^{n_{N}}\left(l_{N}\right)
\end{array}\right)
$$

where $H_{n}$ are the Hermite polynomials for a single harmonic oscillator. The ground state $\Psi_{0}$ corresponds to $n_{1}=0, n_{2}=1, \ldots n_{N}=N-1$. Therefore the generic excited state can be represented by means of a Young diagram with rows $\left(r_{1}, r_{2}, \ldots, r_{N}\right)$, with $r_{i}=n_{i}-i+1$ not all vanishing natural numbers in decreasing order. The energy of the state above the Fermi sea is $E=J=\sum_{i} r_{i}$, which is the total number of boxes in the Young diagram (for the relation to Fermi systems, see [12, 11, 14, 16, 17, 20]).

Let us list a few states which will be considered in the sequel by means of their Young diagram representation. A giant graviton is represented by a single column Young diagram, whose maximum length is of course $N$. A giant graviton, [5, 6, 15] is a half-BPS state which can be described as a D3-brane wrapping around an $S^{3}$ cycles in the $S^{5}$ factor of $A d S_{5} \times S^{5}$. Stability is guaranteed by the spinning of the brane around an axis in $S^{5}$. The angular momentum has an upper bound $J \leq N$, which is a manifestation of the stringy exclusion principle. Since $\Delta=J$, the representation by means of a Young tableau incorporates in a simple way the exclusion principle. 
A dual giant graviton, i.e. a D3-brane wrapping around an $S^{3}$ cycle in $A d S_{5}$, is represented by a one-row Young diagram of arbitrary length (no bound here). A black ring is represented by a large rectangular diagram of size $N$ (see below). A superstar is represented by a large triangular diagram of size $\sim N$. It represents a stack of giant gravitons located at the origin of $A d S_{5}$. From the supergravity viewpoint, it is a singular solution in that it has a naked singularity. It is conjectured that due to string corrections it may actually be completely regular solution.

In the last two cases the energy of the states is proportional to the area of the Young tableau and therefore $\sim N^{2}$. Following in particular [2], these are the states we will be mostly interested in in the following and we will consider them in the large $N$ limit. In [2] a limit shape was introduced for the corresponding Young tableaux in the continuous limit. This is a function $y(x)$, where $x$ runs from left to right along the rows and $y$ from bottom to top along the columns. The origin is set at the leftmost bottom box of the tableau. For instance, for the superstar ensemble we have $\Delta=N N_{c} / 2$ and $y(x)=\frac{N_{c}}{N} x$, where $N_{c}$ is the number of columns.

\section{$2.1 \quad 1 / 2-B P S$ states as supergravity solutions}

In 12 a beautiful characterization of $1 / 2-$ BPS states in type IIB supergravity was found. Regular 1/2-BPS solutions with a geometry invariant under $S O(4) \times S O(4) \times R$ correspond to the following ansatz

$$
\begin{aligned}
d s^{2} & =-h^{-2}\left(d t+V_{i} d x^{i}\right)^{2}+h^{2}\left(d y^{2}+d x^{i} d x^{i}\right)+y e^{G} d \Omega_{3}^{2}+y e^{-G} d \tilde{\Omega}_{3}^{2} \\
h^{-2} & =2 y \cosh G \\
y \partial_{y} V_{i} & =\epsilon_{i j} \partial_{j} z, \quad y\left(\partial_{i} V_{j}-\partial_{j} V_{i}\right)=\epsilon_{i j} \partial_{y} z \\
z & =\frac{1}{2} \tanh G
\end{aligned}
$$

where $i, j=1,2$ and $\epsilon_{i j}$ is the antisymmetric symbol. There are also $N$ units of 5 -form flux, with

$$
F_{(5)}=F_{\mu \nu} d x^{\mu} \wedge d x^{\nu} \wedge d \Omega_{3}+\tilde{F}_{\mu \nu} d x^{\mu} \wedge d x^{\nu} \wedge d \tilde{\Omega}_{3}
$$

where $\mu, \nu=0, \ldots, 3$ refer to $t, x^{1}, x^{2}, y$. As for the ansatz for $F$ and $\tilde{F}$, see [12]. The full solution is determined in terms of a single function $z$, which must satisfy the equation

$$
\partial_{i} \partial_{i} z+y \partial_{y}\left(\frac{\partial_{y} z}{y}\right)=0
$$

One can solve this equation by remarking its analogy with the Laplace equation for an electrostatic potential. Regular solutions can exist only if at the boundary $y=0$ the function $z\left(0, x_{1}, x_{2}\right)$ takes the values $\pm \frac{1}{2}$. Therefore regular solutions correspond to boundary functions $z\left(0, x_{1}, x_{2}\right)$ that are locally constant in the $x_{1}, x_{2}$ plane. The region of this plane where $z=-1 / 2$ are called droplets and denoted by $\mathcal{D}$. Following [2] we reintroduce in the game $\hbar$ and make the identification $\hbar \leftrightarrow 2 \pi \ell_{p}^{4}$, noticing that $x_{1}, x_{2}$ have the unusual dimension of a length square. 
The area of the droplet must equal $N$ :

$$
N=\int_{\mathcal{D}} \frac{d^{2} x}{2 \pi \hbar}
$$

while the conformal dimension of the state corresponding to the droplet $\mathcal{D}$ is

$$
\Delta=\int_{\mathcal{D}} \frac{d^{2} x}{2 \pi \hbar} \frac{1}{2} \frac{x_{1}^{2}+x_{2}^{2}}{\hbar}-\frac{1}{2}\left(\int_{\mathcal{D}} \frac{d^{2} x}{2 \pi \hbar}\right)^{2}
$$

In conclusion, the information about the solution is encoded in the droplet. For instance, if the droplet is a disk of radius $r_{0}$ we recover the $A d S_{5} \times S^{5}$ solution; if the droplet is the upper half plane one gets the plane wave solution. In general if the droplet is compact the solution is asymptotically $A d S_{5} \times S^{5}$. It is useful to introduce the new notation $u\left(0 ; x_{1}, x_{2}\right)=$ $\frac{1}{2}-z\left(0 ; x_{1}, x_{2}\right) ; u$ is the characteristic function of the droplet, since it equals 1 inside the droplet and 0 outside. Solutions with such (sharp) characteristic functions are regular since the boundary conditions are satisfied. Solutions characterized by a function $u$ which is not exactly 1 or 0 , are singular [27, 28] (for a connection with quantum Hall effect, see [21, 22, 19]). This is the case of the superstar solution [8].

\subsection{The Wigner distribution}

It is clearly of upmost importance to establish a dictionary between the $1 / 2-$ BPS states introduced at the beginning of this section starting from $N=4 \mathrm{SYM}$ and the droplet solutions. This is tantamount to finding a recipe to recognize the geometry emerging from a given gauge field theory state. The clue is the free fermion representation introduced above: any state represented by a Young diagram can be interpreted as a system of $\mathrm{N}$ fermions with energies above the Fermi sea. To this end it is useful to rewrite the formulas (51) and (6) in the more general form

$$
\begin{aligned}
\Delta & =\int \frac{d^{2} x}{2 \pi \hbar} \frac{1}{2} \frac{x_{1}^{2}+x_{2}^{2}}{\hbar} u\left(0 ; x_{1}, x_{2}\right)-\frac{1}{2}\left(\int \frac{d^{2} x}{2 \pi \hbar} u\left(0 ; x_{1}, x_{2}\right)\right)^{2} \\
N & =\int \frac{d^{2} x}{2 \pi \hbar} u\left(0 ; x_{1}, x_{2}\right) .
\end{aligned}
$$

where the integration extends over the whole $x_{1}, x_{2}$ plane. These formulas suggest that $u$ be identified with the semiclassical limit of the quantum one-particle $(q, p)$ phase-space distributions of the free dual fermions after the identification $\left(x_{1}, x_{2}\right) \leftrightarrow(q, p)$. A phasespace distribution may be understood as an attempt of assigning a probability distribution to the phase-space points. It is a heuristic concept and there is no unique prescription for it. The most well-known distribution is the Wigner one [24]:

$$
W(q, p)=\frac{1}{2 \pi \hbar} \int_{-\infty}^{\infty} d y\langle q-y|\hat{\rho}| q+y\rangle e^{2 i p y / \hbar}
$$

where $\hat{\rho}$ is the density matrix. In the case of a pure state $\psi,\left\langle q^{\prime}|\hat{\rho}| q^{\prime \prime}\right\rangle=\psi\left(q^{\prime}\right) \psi^{\star}\left(q^{\prime \prime}\right)$, therefore

$$
W(q, p)=\frac{1}{2 \pi \hbar} \int_{-\infty}^{\infty} d y \psi^{\star}(q+y) \psi(q-y) e^{2 i p y / \hbar}
$$


In general $\hat{\rho}$ will take the form of

$$
\hat{\rho}\left(q^{\prime}, q^{\prime \prime}\right)=\sum_{f \in \mathcal{F}} \psi_{f}\left(q^{\prime}\right) \psi_{f}^{\star}\left(q^{\prime \prime}\right)
$$

$\mathcal{F}$ being a given family of pure states. We will consider family of pure states representing excited states of $N$ (fermionic) harmonic oscillators $f_{n}=r_{n}+n-1$, with $n=1, \ldots, N$ (where we have dropped $\hbar$ ). In this case $\mathcal{F}$ will be a subset of the natural numbers and

$$
\psi_{f_{n}}=A\left(f_{n}\right) H_{f_{n}}(q / \sqrt{\hbar}) e^{-q^{2} / 2 \hbar}
$$

where $A(n)$ is a normalization constant and $H_{n}$ are, as above, the Hermite polynomials. Using a well-known integration formula for Hermite polynomials one gets, 24],

$$
W(q, p)=\sum_{f_{n} \in \mathcal{F}} W_{f_{n}}(q, p)=\frac{1}{2 \pi \hbar} e^{-\left(q^{2}+p^{2}\right) / \hbar} \sum_{f_{n} \in \mathcal{F}}(-1)^{f_{n}} L_{f_{n}}\left(2 \frac{q^{2}+p^{2}}{\hbar}\right)
$$

Thinking of $W$ as a probability distribution is certainly a heuristic and approximate concept, because it may be negative (see the considerations in [2, where an improved always positive distribution is introduced, the Husimi distribution). However we will not need it in the following, because we will compare Wigner distributions with space profiles of VSFT (which are not probability distributions either).

Here we are interested in Wigner distributions because they represent a precise recipe to bosonize associated fermion systems: from the fermion system we easily get the Wigner distribution and from the latter we can reconstruct the former, 16, 23. In the following we will use Wigner distributions in this sense, and will be concerned specifically with distributions relative to ensembles, in which $N$ is supposed to be very large. The semiclassical limit will correspond to $\hbar \rightarrow 0$ keeping $\hbar N$ finite. We will use such distributions to make a comparison with the $u$ droplet distributions, [2], and with space profiles in VSFT (for coarse-graining, see also [13, 4, 26]).

Let us consider a few significant cases. The first concerns the Fermi sea. The relevant distribution is

$$
2 \pi \hbar W_{F S}=2 \pi \hbar \sum_{n=0}^{N-1} W_{n}(q, p)
$$

By using a well-known identity for Laguerre polynomials one formally obtains 1 when the summation extends to infinity with fixed $\hbar$. This would not correspond to $A d S_{5} \times S^{5}$. However a numerical analysis shows that the limit $\hbar \rightarrow 0$ with $\hbar N$ fixed reproduces the finite disk characteristic of the latter solution (see, for instance, 25]).

The second example involves the Young diagram corresponding to a giant graviton. It has $r_{n}=0, n<k$ and $r_{n}=1$ for $k \leq n \leq N$. The distribution is

$$
2 \pi \hbar W_{G G}=2 \pi \hbar\left(\sum_{n=0}^{k-2}+\sum_{n=k}^{N}\right) W_{n}(q, p)
$$

It is evident that in the large $N$ limit with $k$ fixed, this distribution will be indistinguishable from the Fermi sea one. 
The third example is the case corresponding to a rectangular Young diagram of row length $K$. It represents $N$ fermions all excited above the sea by the same amount $K$. There is no a priori relation between $N$ and $K$, but we are interested in the limit of large $N$ and $K$ such that $\hbar K$ as well as $\hbar N$ are finite. The Wigner distribution is

$$
2 \pi \hbar W_{\text {rect }}=2 \pi \hbar \sum_{n=K}^{N+K-1} W_{K+n-1}(q, p)
$$

Setting $u\left(0, x_{1}, x_{2}\right)=2 \pi \hbar W_{\text {rect }}$ this identifies a characteristic function which is (approximately) 1 in the ring $\hbar K \leq \frac{q^{2}+p^{2}}{2} \leq \hbar(N+K)$ and 0 outside, in the large $N$ and $K$ limit. This corresponds to the $1 / 2-$ BPS called "black ring" in [12. It has conformal dimension $\Delta=N K \sim N^{2}$, since $K$ must be some rational multiple of $N$.

The last example concerns Young diagrams which are approximately triangular with $\Delta=N N_{c} / 2$ and so correspond to superstar ensembles. In this case we have $f_{n}=(n-1) \delta_{n}$, with $\delta_{n}$ an integer $\sim \frac{N_{c}}{N}+1$. For illustrative purposes let us set $\delta_{n}=\delta=\frac{N_{c}}{N}+1$. Then

$$
2 \pi \hbar W_{\text {triangle }}=2 \pi \hbar \sum_{n=0}^{N-1} W_{n \delta}(q, p)=2 e^{-\frac{2 H}{\hbar}} \sum_{n=0}(-1)^{n \delta} L_{n \delta}(4 H / \hbar)
$$

where $H=\left(q^{2}+p^{2}\right) / 2$. The result of the analysis in [2] is that in the large $N$ limit

$$
2 \pi \hbar W_{\text {triangle }}^{\infty}=\frac{1}{\delta}+\text { oscillations at scale } \Delta \mathrm{H}=\hbar
$$

Therefore identifying once again $2 \pi \hbar W_{\text {triangle }}^{\infty}$ with $u\left(0 ; x_{1}, x_{2}\right)$ we get approximately $u\left(0, x_{1}, x_{2}\right)=$ $1 / \delta$ within a finite radius disk. This corresponds to a fractionally filled droplet and represents the superstar solution, which is singular. It is also an explicit example of the relations

$$
u\left(0 ; r^{2}\right)=\frac{1}{1+y^{\prime}}=g(E)
$$

which was conjectured and verified in various examples in 2]. The function $g(E)$ is called grayscale distribution and encodes the effective behavior of coarse-grained semiclassical observables in a given quantum state.

\section{VSFT: a reminder}

In this section we recall what is strictly necessary from VSFT in order to render this paper self-contained. The VSFT action is

$$
\mathcal{S}(\Psi)=-\left(\frac{1}{2}\langle\Psi|\mathcal{Q}| \Psi\rangle+\frac{1}{3}\langle\Psi \mid \Psi * \Psi\rangle\right)
$$

where

$$
\mathcal{Q}=c_{0}+\sum_{n>0}(-1)^{n}\left(c_{2 n}+c_{-2 n}\right)
$$


The ansatz for nonperturbative solutions is in the factorized form

$$
\Psi=\Psi_{m} \otimes \Psi_{g}
$$

where $\Psi_{g}$ and $\Psi_{m}$ depend purely on ghost and matter degrees of freedom, respectively. The equation of motion splits into

$$
\begin{aligned}
\mathcal{Q} \Psi_{g} & =-\Psi_{g} *_{g} \Psi_{g} \\
\Psi_{m} & =\Psi_{m} *_{m} \Psi_{m}
\end{aligned}
$$

where $*_{g}$ and $*_{m}$ refer to the star product involving only the ghost and matter part. The action for this type of solution is

$$
\mathcal{S}(\Psi)=-\frac{1}{6}\left\langle\Psi_{g}|\mathcal{Q}| \Psi_{g}\right\rangle\left\langle\Psi_{m} \mid \Psi_{m}\right\rangle
$$

$\left\langle\Psi_{m} \mid \Psi_{m}\right\rangle$ is the ordinary inner product. We will first concentrate on the matter part, eq.(21), assuming the existence of a universal ghost solution. The solutions are projectors of the $*_{m}$ algebra. The $*_{m}$ product is defined as follows

$$
{ }_{123}\left\langle V_{3} \mid \Psi_{1}\right\rangle_{1}\left|\Psi_{2}\right\rangle_{2}={ }_{3}\left\langle\Psi_{1} *_{m} \Psi_{2}\right|,
$$

see [31, 32, 33, 34] for the definition of the three string vertex ${ }_{123}\left\langle V_{3}\right|$. The basic ingredient in this definition are the matrices of vertex coefficients $V_{n m}^{r s}, r, s=1,2,3, n, m=1, \ldots, \infty$.

Now we look for solutions that mimic the behavior of the half-BPS states discussed in the previous section. As it turns out they must be superpositions of matter projectors (stacks of VSFT D-branes). The latter have the following characteristics: they must cover the ordinary 4D Minkowski space (parallel directions), be, in the low energy limit $\left(\alpha^{\prime} \rightarrow 0\right)$, delta-function like in 16 directions and have some width in the remaining 6 directions (these 22 directions will be referred to as the transversal ones). Out of the latter two will have a special status, in that a constant $B$ field will be switched on along them. We can imagine that all the internal dimensions are compactified on tori, but this is not strictly necessary for our argument. In the remaining part of this section we deal with the construction of the solutions and postpone until the next section a discussion of their connection with the previous section.

In the following we need both translationally invariant (in the parallel directions) and non-translationally invariant solutions (in the transverse directions).

Although there is a great variety of such solutions we will stick to those introduced in 35], i.e. the sliver and the lump. The former is translationally invariant and is defined by

$$
|\Xi\rangle=\mathcal{N} e^{-\frac{1}{2} a^{\dagger} \cdot S \cdot a^{\dagger}}|0\rangle, \quad a^{\dagger} \cdot S \cdot a^{\dagger}=\sum_{n, m=1}^{\infty} a_{n}^{\mu \dagger} S_{n m} a_{m}^{\nu \dagger} \eta_{\mu \nu}
$$

where $S=C T$ and

$$
T=\frac{1}{2 X}(1+X-\sqrt{(1+3 X)(1-X)})
$$

with $X=C V^{11}$, where $C_{n m}=(-1)^{n} \delta_{n m}$ is the so-called twist matrix. The normalization constant $\mathcal{N}$ needs being regularized and is formally vanishing. It has been showed in other 
papers how this problem could be dealt with, [38, 39. Our basic projector will have the form of the sliver along the the space-time directions

The lump solution was engineered to represent a lower dimensional brane (Dk-brane), therefore it will have $(25-k)$ transverse space directions along which translational invariance is broken. Accordingly we split the three string vertex into the tensor product of the perpendicular part and the parallel part

$$
\left|V_{3}\right\rangle=\left|V_{3, \perp}\right\rangle \otimes\left|V_{3, \|}\right\rangle
$$

The parallel part is the same as in the sliver case while the perpendicular part is modified as follows. Following [35], we denote by $x^{\bar{\mu}}, p^{\bar{\mu}}, \bar{\mu}=1, \ldots, k$ the coordinates and momenta in the transverse directions and introduce the canonical zero modes oscillators

$$
a_{0}^{(r) \bar{\mu}}=\frac{1}{2} \sqrt{b} \hat{p}^{(r) \bar{\mu}}-i \frac{1}{\sqrt{b}} \hat{x}^{(r) \bar{\mu}}, \quad a_{0}^{(r) \bar{\mu} \dagger}=\frac{1}{2} \sqrt{b} \hat{p}^{(r) \bar{\mu}}+i \frac{1}{\sqrt{b}} \hat{x}^{(r) \bar{\mu}},
$$

where $b$ is a free parameter. Denoting by $\left|\Omega_{b}\right\rangle$ the oscillator vacuum $\left(a_{0}^{\bar{\mu}}\left|\Omega_{b}\right\rangle=0\right)$, in this new basis the three string vertex is given by

$$
\left|V_{3, \perp}\right\rangle^{\prime}=K e^{-E^{\prime}}\left|\Omega_{b}\right\rangle
$$

$K$ being a suitable constant and

$$
E^{\prime}=\frac{1}{2} \sum_{r, s=1}^{3} \sum_{M, N \geq 0} a_{M}^{(r) \bar{\mu} \dagger} V_{M N}^{\prime r s} a_{N}^{(s) \bar{\nu} \dagger} \eta_{\bar{\mu} \bar{\nu}}
$$

where $M, N$ denote the couple of indices $\{0, m\}$ and $\{0, n\}$, respectively. The coefficients $V_{M N}^{\prime r s}$ are given in Appendix B of [35]. The new Neumann coefficients matrices $V^{\prime r s}$ satisfy the same relations as the $V^{r s}$ ones. In particular one can introduce the matrices $X^{\prime r s}=$ $C V^{\prime} r s$, where $C_{N M}=(-1)^{N} \delta_{N M}$. The lump solution $\left|\Xi_{k}^{\prime}\right\rangle$ has the form (24) with $S$ along the parallel directions, while $|0\rangle$ is replaced by $\left|\Omega_{b}\right\rangle$ and $S$ is replaced by $S^{\prime}$ along the perpendicular ones. Here $S^{\prime}=C T^{\prime}$ and $T^{\prime}$ has the same form as $T$ eq. (25) with $X$ replaced by $X^{\prime}$. The normalization constant $\mathcal{N}^{\prime}$ is defined in a way analogous to $\mathcal{N}$. It can be verified that the ratio of tensions for such solutions is the appropriate one for $D k$-branes. For our basic projector we will choose $k=22$.

As said above, two of the transverse directions are special, in that a constant background $B$ field is switched on there. We denote these two directions by the labels $\alpha$ and $\beta$ (for instance $\alpha, \beta=24,25)$ and denote them simply by $y_{1}, y_{2}$; we take for $B$ the explicit form

$$
B_{\alpha \beta}=\left(\begin{array}{cc}
0 & B \\
-B & 0
\end{array}\right)
$$

Then, as is well-known [45, 48, 49, in these two directions we have a new effective metric $G_{\alpha \beta}$, the open string metric, as well as an effective antisymmetric parameter $\theta_{\alpha \beta}$, given by

$$
G_{\alpha \beta}=\sqrt{\operatorname{Det} G} \delta_{\alpha \beta}, \quad \theta^{\alpha \beta}=-\epsilon^{\alpha \beta} \theta
$$


where until further notice we set $\alpha^{\prime}=1$ and $\operatorname{Det} G=\left(1+(2 \pi B)^{2}\right)^{2}$. In the low energy limit $\theta \sim 1 / B$. In (31) $\epsilon^{\alpha \beta}$ represents the $2 \times 2$ antisymmetric symbol with $\epsilon^{1}{ }_{2}=1$. The transverse vertex $\left|V_{3, \perp}\right\rangle$ will become in this case $\left|V_{3, \perp}^{\prime}\right\rangle$

$$
\left|V_{3, \perp}^{\prime}\right\rangle=\left|V_{3, \perp, \theta}\right\rangle \otimes\left|V_{3, \perp}\right\rangle
$$

where

$$
\left|V_{3, \perp, \theta}\right\rangle=K_{\theta} e^{-E_{\theta}}\left|\Omega_{b}\right\rangle
$$

$K_{\theta}$ is a suitable constant and, [42, 33],

$$
E_{\theta}=\frac{1}{2} \sum_{r, s=1}^{3} \sum_{M, N \geq 0} a_{M}^{(r) \alpha \dagger} \mathcal{V}_{\alpha \beta, M N}^{r s} a_{N}^{(s) \beta \dagger}
$$

The coefficients $\mathcal{V}_{M N}^{\alpha \beta, r s}$ are given in [42]. The new Neumann coefficients matrices $\mathcal{V}^{\text {rs }}$ satisfy the same relations as the $V^{r s}$ ones. One introduces the matrices $X^{r s}=C V^{r s}$. Then the lump solution $|\mathcal{S}\rangle$ along $\alpha$ and $\beta$ has the form (24) with $|0\rangle$ replaced by $\left|\Omega_{b}\right\rangle$ and $S$ replaced by $\mathcal{S}$, where $\mathcal{S}=C \mathcal{T}$ and $\mathcal{T}$ has the same form as $T$ in eq.(25) with $X$ replaced by $X$. It can be verified that the ratio of tensions for such solutions is the appropriate one for $D$-branes in a magnetic field, 43 .

It is possible to construct a full family of such solutions which are $*-$ and $b p z-$ orthonormal. This goes as follows, 44, 33. First we introduce two 'vectors' $\xi=\left\{\xi_{N \alpha}\right\}$ and $\zeta=\left\{\zeta_{N \alpha}\right\}$, which are chosen to satisfy the conditions

$$
\rho_{1} \xi=0, \quad \rho_{2} \xi=\xi, \quad \text { and } \quad \rho_{1} \zeta=0, \quad \rho_{2} \zeta=\zeta,
$$

where $\rho_{1}, \rho_{2}$ are the half-string projectors [36, 37]. Moreover we define the matrix $\tau$ as $\tau=\left\{\tau_{\alpha}^{\beta}\right\}=\left(\begin{array}{cc}1 & 0 \\ 0 & -1\end{array}\right)$. Next we set

$$
\mathrm{x}=\left(a^{\dagger} \tau \xi\right)\left(a^{\dagger} C \zeta\right)=\left(a_{N}^{\alpha \dagger} \tau_{\alpha}^{\beta} \xi_{N \beta}\right)\left(a_{N}^{\alpha \dagger} C_{N M} \zeta_{M \alpha}\right)
$$

Finally we introduce the Laguerre polynomials $L_{n}(z)$, of the generic variable $z$, and define the sequence of states

$$
\left|\Lambda_{n}\right\rangle=(-\kappa)^{n} L_{n}\left(\frac{\mathrm{x}}{\kappa}\right)\left|\mathcal{S}_{\perp \theta}\right\rangle
$$

where, for simplicity, we have written down the tensorial factor involving the the $y_{1}, y_{2}$ directions only and understood the other directions. As part of the definition of $\left|\Lambda_{n}\right\rangle$ we require the two following conditions to be satisfied

$$
\xi^{T} \tau \frac{1}{\mathbb{I}-\mathcal{T}^{2}} \zeta=1, \quad \xi^{T} \tau \frac{\mathcal{T}}{\mathbb{I}-\mathcal{T}^{2}} \zeta=\kappa
$$

where $\kappa$ is a real number. To guarantee Hermiticity for $\left|\Lambda_{n}\right\rangle$, we require

$$
\zeta=\tau \xi^{*} .
$$


The states $\left|\Lambda_{n}\right\rangle$ satisfy the remarkable property

$$
\begin{aligned}
& \left|\Lambda_{n}\right\rangle *\left|\Lambda_{m}\right\rangle=\delta_{n, m}\left|\Lambda_{n}\right\rangle \\
& \left\langle\Lambda_{n} \mid \Lambda_{m}\right\rangle=\delta_{n, m}\left\langle\Lambda_{0} \mid \Lambda_{0}\right\rangle
\end{aligned}
$$

Therefore each $\Lambda_{n}$, as well as any combination of $\Lambda_{n}$ with unit coefficients, are lump solution.

So far we have set $\alpha^{\prime}=1$. It is easy to insert back $\alpha^{\prime}$. In order to evaluate the low energy profile of $\left|\Lambda_{n}\right\rangle$ we first contract it with the eigenstate of the position operators with eigenvalues $y^{\alpha}:\left\langle y \mid \Lambda_{n}\right\rangle$, and then take the limit $\alpha^{\prime} \rightarrow 0$, 41, 43, 44. The leading term in the $\alpha^{\prime}$ expansion turns out to be

$$
\left\langle y \mid \Lambda_{n}\right\rangle=\frac{1}{\pi}(-1)^{n} L_{n}\left(\frac{2 \rho^{2}}{\theta}\right) e^{-\frac{\rho^{2}}{\theta}}|\Xi\rangle+\mathcal{O}\left(\sqrt{\alpha^{\prime}}\right)
$$

where $\rho^{2}=y^{\alpha} y^{\beta} \delta_{\alpha \beta}$ and $|\Xi\rangle$ is the sliver solution.

The projectors we need in the following have this $\alpha^{\prime} \rightarrow 0$ limit in the $y^{\alpha}$ directions; as for the remaining directions, they have the form of the sliver in the parallel directions and, finally, they become delta-like functions multiplied by the sliver in the remaining transverse directions, i.e. they are localized at the origin of the latter. This can be easily seen by taking the limit $\theta \rightarrow 0$ in the case $n=0$ in $(42){ }^{6}$.

\section{A correspondence}

Looking at eqs.121415) of section 2, one immediately notices that they can be seen (up to an overall normalization constant) as the low energy limit space profiles of combinations of the string states $\Lambda_{n}$ introduced in the previous section, with unit coefficients. Since combinations of $\Lambda_{n}$ with unit coefficients are solutions to the equation of motion of VSFT, we can see the above Wigner distributions as the low energy profile of VSFT solutions (up to the common $|\Xi\rangle$ factor). It is therefore tantalizing to make the following association

\section{Wigner distribution for an $N$ fermion system $\leftrightarrow$ VSFT solution}

For this to work we must require the correspondence ${ }^{7} \hbar \leftrightarrow \theta$ and that the coordinates $x_{1}, x_{2}$ be identified with $y_{1}, y_{2}$. This is what we suggest and would like to motivate in this section. The previous correspondence can be read in two directions. First: one can say that to any $1 / 2$-BPS state to which we can associate a Wigner distribution of the type (9), there corresponds a VSFT solution given by a combination

$$
\left|W_{\mathcal{F}}\right\rangle=\sum_{f_{n} \in \mathcal{F}}\left|\Lambda_{f_{n}}\right\rangle, \quad 2 \hbar W(q, p)|\Xi\rangle=\langle y \mid W\rangle
$$

where $(p, q)$ is identified with $\left(y_{1}, y_{2}\right)$ and the latter are the eigenvalues of $|y\rangle$. Vice versa: to any VSFT solution of the type (43) we can associate a Wigner distribution $W(q, p)$

\footnotetext{
${ }^{6}$ One could easily construct projectors that are 'fat' also along other transverse directions, but we will not need them in the sequel.

${ }^{7}$ It should be recalled that on the SUGRA side we have three parameters, $\alpha^{\prime}, g_{s}$ and $N$. With the first two one forms the combination $\hbar=2 \pi g_{s} \alpha^{\prime 2}$. On the VSFT side we have also three parameters $\alpha^{\prime}, \theta$ and $N$.
} 
according to (91). In this way we can associate to $|W\rangle$ a Young tableau and therefore a $1 / 2$-BPS state in the $\mathcal{N}=4$ superconformal field theory (before taking the large $N$ limit) and we can associate a geometry (after taking it ${ }^{8}$ ). The latter point of view is probably the most interesting one. It implies that we may be able to associate a geometry to a given VSFT solution, therefore we are in the condition to answer some of the questions posed by open-closed string duality. Here we see how geometry emerges from a VSFT solution which is entirely expressed in terms of open string creation operators.

In the following we would like to list some arguments in support of our proposal.

1) With the above association we connect a microstate corresponding to a geometry, which is a supergravity solution, to a string state which is a solution of the VSFT equation of motion. The correspondence (43) is one-to-one ${ }^{9}$ (before the large $N$ limit).

2) The droplet geometry lives in a $\left(x_{1}, x_{2}\right)$ plane which lies in the internal (compactified) dimensions. In the same way the plane $\left(y_{1}, y_{2}\right)$ stays in the compactified part of the bosonic target space. As pointed out above, we identify the two planes. One could phrase it by saying that the two space coordinates $x_{1}, x_{2}$, which had been replaced by two phase-space coordinates $q, p$ in the intermediate argument, have returned to their natural role via the identification with $y_{1}, y_{2}$.

3) The correspondence (43) tells us how the Pauli principle gets incorporated into a bosonic setting. The numbers $f_{n}$ in the LHS of (43) correspond to the fermion energy levels in the original fermion system. Therefore, due to the Pauli exclusion principle, each $f_{n}$ can appear only once in the family $\mathcal{F}$. Therefore in the summation each $\left|\Lambda_{f_{n}}\right\rangle$ appears only once. This guarantees that $|W\rangle$ is a VSFT solution ${ }^{10}$. On the other hand any VSFT solution that can be written in the form $\sum_{f_{n} \in \mathcal{F}}\left|\Lambda_{f_{n}}\right\rangle$ tells us that the numbers $f_{n} \in \mathcal{F}$ can be interpreted as energy levels of a fermionic harmonic oscillator system, since each appears only once. This is the way the D-brane solutions of VSFT manifest their fermionic nature.

4) The VSFT solution corresponding to the Fermi sea (12) is represented by a stack of $N$ (unstable) VSFT D-branes. The giant graviton solution (13) is represented by a D-brane missing from the stack. The superstar solution (15) is represented by a stack of such missing (unstable) D-branes. This is in keeping with the interpretation of superstars as stack of giant gravitons, 8]. (It is worth remarking at this point that all the VSFT solutions we consider in this paper are composite of VSFT D-branes and there is no direct identification between single VSFT D-branes and single D3-branes in superstring theory.)

5) There is an algebra isomorphism between Wigner distributions of the type (9) and VSFT solutions like $|W\rangle$, an isomorphism that was pointed out in [46, 47, 44]. It is a wellknown fact that any classical function $f(q, p)$ in a $(q, p)$ phase space can be mapped to a quantum operator $\hat{O}_{f}$ via the Weyl transform, and that the product for quantum operators $\hat{O}_{f} \hat{O}_{g}$ is mapped into the Moyal product $f \star g$ for functions. Under this correspondence the $\left(x_{1}, x_{2}\right) \leftrightarrow(q, p)$ plane becomes noncommutative. It is a well-known fact that, under this correspondence the classical Wigner distributions like (121415) are mapped into projectors of the Moyal star algebra:

$$
(2 \pi \hbar W) \star(2 \pi \hbar W)=2 \pi \hbar W .
$$

\footnotetext{
${ }^{8}$ In the process of taking the large $N$ limit one smears out many details, so that multiple states are mapped to the same geometry

${ }^{9}$ See on this point the remark at the end of section 5 .

${ }^{10}$ If $|\Lambda\rangle$ is some $*$-projector, $n|\Lambda\rangle$ is a $*-$ projector if and only if $n=0,1$.
} 
Actually these distributions turn out to coincide with families of the so-called GMS solitons, [51, 50. Let us recall the relevant construction. Define the harmonic oscillator $a=(\hat{q}+$ $i \hat{p}) / \sqrt{(2 \theta)}$ and its hermitian conjugate $a^{\dagger}:\left[a, a^{\dagger}\right]=1$. The normalized harmonic oscillator eigenstates are: $|n\rangle=\frac{\left(a^{\dagger}\right)^{n}}{\sqrt{n} !}|0\rangle$. Now, via the Weyl correspondence, we can map any rank one projector $|n\rangle\langle n|$ to a classical function of the coordinates $x_{1}, x_{2}$.

$$
|n\rangle\langle n| \longleftrightarrow \psi_{n}\left(x_{1}, x_{2}\right)=2(-1)^{n} L_{n}\left(\frac{2 r^{2}}{\theta}\right) e^{-\frac{r^{2}}{\theta}}
$$

where $r^{2}=x_{1}^{2}+x_{2}^{2}$. Each of these solutions, by construction, satisfy $\psi_{n} \star \psi_{n}=\psi_{n}$. These are referred to as GMS solitons [51]. In the previous section we have shown that the low energy limit of $\left\langle y \mid \Lambda_{n}\right\rangle$ factorizes into the product of the sliver state and $\psi_{n}\left(y_{1}, y_{2}\right)$. This means that the VSFT star product factorizes into Witten's star product and the Moyal $\star$ product, 46, 47. More precisely we can formalize the following isomorphism

$$
\begin{aligned}
& \left|\Lambda_{n}\right\rangle \quad \longleftrightarrow P_{n} \longleftrightarrow \psi_{n}\left(y_{1}, y_{2}\right) \\
& \left|\Lambda_{n}\right\rangle *\left|\Lambda_{n^{\prime}}\right\rangle \longleftrightarrow P_{n} P_{n^{\prime}} \longleftrightarrow \psi_{n} \star \psi_{n^{\prime}}
\end{aligned}
$$

where $\star$ denotes the Moyal product.

This remark should not be underestimated. Let us consider $2 \pi \hbar W$ and suppose it is such that we can ignore its derivatives with respect to $p$ and $q$. Then eq. (444) becomes $(2 \pi \hbar W)^{2}=2 \pi \hbar W$, which is the equation of a characteristic function (it can only be either 0 or 1 ). This is indeed what happens in the case of the vacuum and the black ring solutions, see 2. 3]. It is not the case of the superstar distribution because in that case we cannot ignore derivatives. But this remark suggests that the property of being Moyal projectors is basic for Wigner distributions to represent 1/2-BPS states. The string state $|W\rangle$ 'inherits' this property, it is the 'continuation' of the space profile to the whole string theory. In this sense it is natural that $|W\rangle$ be a string field theory solution.

6) Finally one should point out that there exists a solution generating technique that allows one to produce new solutions starting from a fixed one. As an example let us consider the partial isometries introduced in [52]. They are defined as follows

$$
\begin{aligned}
& \left(\mathcal{P}_{+}\right)^{k}\left|\Lambda_{n}\right\rangle=\left|\Lambda_{n+k}\right\rangle \\
& \left(\mathcal{P}_{-}\right)^{k}\left|\Lambda_{n}\right\rangle=\left|\Lambda_{n-k}\right\rangle
\end{aligned}
$$

where we define $\Lambda_{n}=0$ for $n<0$ (see [52 for definitions of $\mathcal{P}_{ \pm}$)). Given a state represented by a certain Young diagram, the operator $\left(\mathcal{P}_{+}\right)^{k}$ adds k boxes in each of the $N$ rows, while its inverse $\left(\mathcal{P}_{-}\right)^{k}$ removes them. Consider for instance the Fermi sea solution corresponding to eq.(12) and apply to it $\left(\mathcal{P}_{+}\right)^{K}$. According the above equations one gets the solution corresponding to the rectangular Young diagram (14). We have seen that (14), in the large $N$ limit, leads to the black ring geometry. Note that the transformations that are generated by such partial isometries are area preserving on the droplet plane (the number of fermions is left unchanged). This points to the fact that partial isometries on the open string side are mapped to topology changing transformations on the closed string side. 


\subsection{Matching observables}

In this subsection we deal with the identification of the quantities in VSFT that correspond to two basic observables in the superconformal field theory and in the supergravity side. The latter are given by the total five form flux, $N$, and by the energy, $\Delta$, (5) 6). We would like to see how these two observables are encoded in the star algebra that characteres the VSFT solutions.

The total flux is simply given by the bpz norm of the projector corresponding to the given Young diagram. We have indeed, (40),

$$
N=\frac{\left\langle W_{\mathcal{F}} \mid W_{\mathcal{F}}\right\rangle}{\left\langle\Lambda_{0} \mid \Lambda_{0}\right\rangle}
$$

This is perfectly expected as the total flux is determined by the number of boundary branes producing it. Note that, differently from the usual open string description given by the gauge theory, this observable is not part of the definition of the theory but is part of a classical solution (in much the same way as it happens in gravity).

In order to understand how the observable corresponding to $\Delta$ emerges from the star algebra, an extension of the (40) is necessary. Consider the following non twist invariant states, 33

$$
\begin{aligned}
\left|\Lambda_{n, m}\right\rangle & =\sqrt{\frac{n !}{m !}}(-\kappa)^{n} Y^{m-n} L_{n}^{m-n}\left(\frac{\mathrm{x}}{\kappa}\right)\left|\mathcal{S}_{\perp \theta}\right\rangle, & & n \leq m \\
\left|\Lambda_{n, m}\right\rangle & =\sqrt{\frac{m !}{n !}}(-\kappa)^{m} X^{n-m} L_{m}^{n-m}\left(\frac{\mathrm{x}}{\kappa}\right)\left|\mathcal{S}_{\perp \theta}\right\rangle, & & n \geq m
\end{aligned}
$$

where

$$
X=a^{\dagger} \tau \xi \quad Y=a^{\dagger} C \zeta
$$

so that $\mathrm{x}=X Y$, and $L_{n}^{m-n}(z)=\sum_{k=0}^{m}\left(\begin{array}{c}m \\ n-k\end{array}\right)(-z)^{k} / k !$ are the generalized Laguerre polynomials. Note that $\Lambda_{n}=\Lambda_{n n}$.

These states star-multiply among themselves in the following way

$$
\Lambda_{n m} * \Lambda_{p q}=\delta_{m p} \Lambda_{n q}
$$

In VSFT they have been shown to be the vacuum states for perturbative strings stretched between the $\mathrm{n}$-th and the $\mathrm{m}$-th brane, 52 .

Thanks to this extended algebra we can explicitly realize the fermionic system of our concern. To this end let's define the following inner operators acting on the string Hilbert space

$$
\begin{aligned}
& A_{+} \phi=\sum_{n=0}^{\infty} \sqrt{n+1} \Lambda_{n+1, n} * \phi * \Lambda_{n, n+1} \\
& A_{-} \phi=\sum_{n=0}^{\infty} \sqrt{n+1} \Lambda_{n, n+1} * \phi * \Lambda_{n+1, n}
\end{aligned}
$$


for any string state $\phi$. These are (the adjoint representation of) the string field oscillators defined in [55] (at fixed half string vector) and behave as the raising/lowering operators of a harmonic oscillator

$$
\begin{aligned}
& A_{+} \Lambda_{n}=\sqrt{n+1} \Lambda_{n+1} \\
& A_{-} \Lambda_{n}=\sqrt{n} \Lambda_{n-1}
\end{aligned}
$$

It is then natural to consider the operator

$$
H=A_{+} A_{-}
$$

which, up to zero point energy, is the analog of the harmonic oscillator hamiltonian.

For single brane states we have

$$
H \Lambda_{n}=n \Lambda_{n}
$$

and, more important,

$$
n=\frac{\left\langle\Lambda_{n}|H| \Lambda_{n}\right\rangle}{\left\langle\Lambda_{0} \mid \Lambda_{0}\right\rangle}
$$

If we evaluate this operator on the stack $W_{\mathcal{F}}$ we get

$$
\frac{\left\langle W_{\mathcal{F}}|H| W_{\mathcal{F}}\right\rangle}{\left\langle\Lambda_{0} \mid \Lambda_{0}\right\rangle}=\sum_{n=0}^{N-1} f_{n}
$$

This is nothing but the energy of the corresponding fermion ensemble given by the Young diagram $\mathcal{F}$.

Now let us define the observable that corresponds to $\Delta$, which we will denote with the same symbol. As in the gravity side $\Delta$ is defined as the difference in 'energy' between the state under consideration and the 'vacuum' (empty AdS), at fixed five form flux $N$, see (6).

$$
\Delta=\frac{\left\langle W_{\mathcal{F}}|H| W_{\mathcal{F}}\right\rangle-\left\langle W_{\mathcal{F}_{0}}|H| W_{\mathcal{F}_{0}}\right\rangle}{\left\langle\Lambda_{0} \mid \Lambda_{0}\right\rangle}=\text { number of boxes of the Young Tableau }
$$

where $\mathcal{F}_{0}=\{0,1, \ldots, N-1\}$ is the Fermi sea at given $N$.

We remark that in open string theory $N$ in (50) is interpreted as an energy, while $\Delta$ in (57) is not (but it can be intepreted as energy in the closed string side under the open-closed string duality, [56]).

\section{Discussion}

The correspondence (43) in the previous section is based on a series of facts, which have been listed above. The coincidence might be accidental, but we tend to believe it has a deeper meaning. The suggestion that comes from the previous section is summarized in the 
following table:

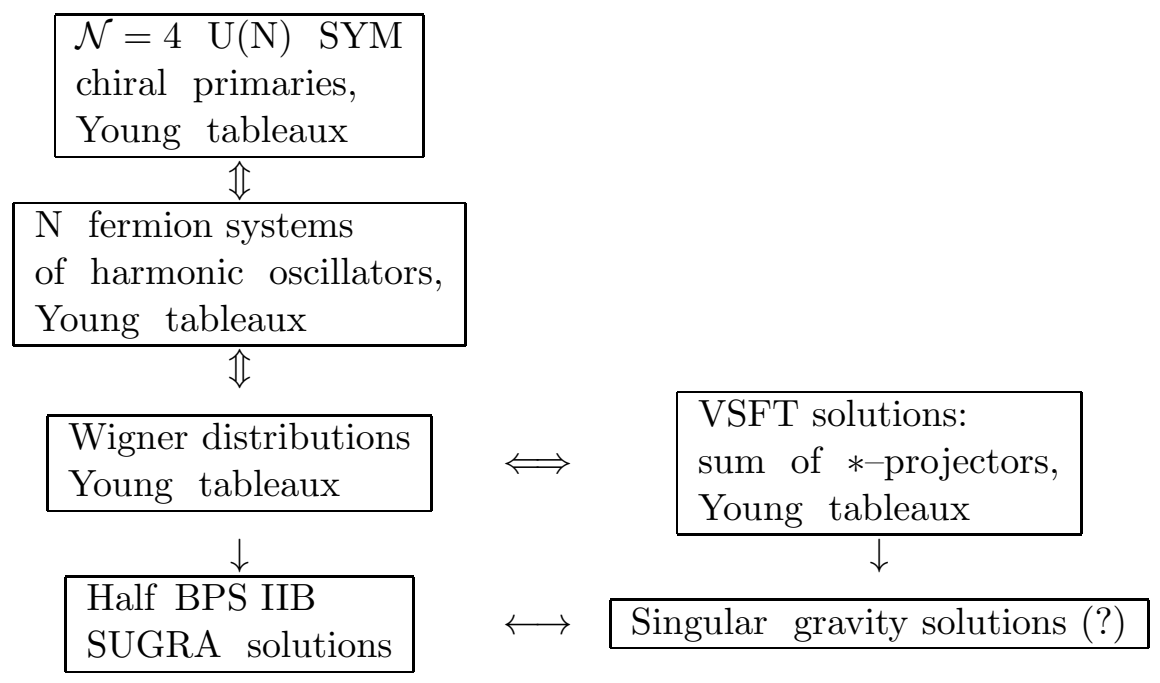

where double-line arrows represent one-to-one correspondences, simple down arrows represent the large $N$ limit and the question mark indicates the conjectural part of our proposed correspondence. Let us describe it in more detail.

The fact that $|W\rangle$ is a VSFT solution is the strongest support of our conjectured correspondence. The weak point is that we know it is a solution of bosonic VSFT but we do not know whether it is a solution of the supersymmetric vacuum string field theory. However we would like to notice that the 1/2-BPS states considered in [1, 2, 3] in the gauge theory side, are all (very heavy) bosonic states. It is not unconceivable that the bosonic part of $1 / 2-$ BPS states is well described by solutions of the bosonic string theory. Unfortunately the study of the tachyon condensation in superstring field theory has not progressed much, see [53, 54]. From what we know nowadays it is possible that the bosonic parts of some solutions of supersymmetric VSFT take a form like $|W\rangle$, although a complete solutions has not yet been determined.

This raises a problem as to the interpretation of the lower right corner of the above table. Based on the above argument, they should represent the bosonic part of supergravity solutions. Now the Einstein equation for the latter is

$$
R_{\mu \nu} \sim F_{\mu l_{1} l_{2} l_{3} l_{4}} F_{\nu}^{l_{1} l_{2} l_{3} l_{4}}
$$

where $F$ is the five-form field strength and where we have set the dilaton to 0 . The contribution of the RHS is basic in the case of LLM solutions, as the latter do not satisfy the pure gravity equation $R_{\mu \nu}=0$. As a consequence the solutions in the lower right corner above are not pure gravity solutions. This is the problem we alluded to above: from a purely bosonic theory we get, in the low energy limit, (the bosonic part of )supergravity solutions. The most likely explanation of this surprising result lies in the type of limit we have taken in VSFT: first $\alpha^{\prime} \rightarrow 0$ with $\theta$ fixed, and then $\theta \rightarrow 0$ with $\theta N$ fixed. This two-step limit selects the (bosonic part of the) droplet-like supergravity solutions. But, on the VSFT side, there are other possible limits. For instance, one could take the same limit, but in three steps, first $\alpha^{\prime} \rightarrow 0$, then $\theta \rightarrow 0$ and finally $N \rightarrow \infty$. This shrinks the droplets to zero size and leads to singular (in the sense of delta-like) solutions, which we can identify with 
(singular) pure gravity solutions. However these solutions are too singular to base on them any serious discussion. So let us deal with this subject from another viewpoint.

From the above we see that the parameter $\theta$, i.e. the $B$ field, plays a fundamental role in the VSFT side limit. There is no $B$ field in the IIB supergravity solutions side, there is instead a background five-form field, whose flux in suitable units equals $N$. Of course in the bosonic SFT side there cannot be any such background. However we have seen that $\theta \sim 1 / B$ is identified with $\hbar$ and, in the large $N$ limit, $\hbar N$ is kept finite. Therefore $B$ and the five-form flux play a parallel role. The five-form flux supports the supergravity solution, while $B$ supports the corresponding VSFT solution, which otherwise would collapse to a delta function. It looks like $B$ is a surrogate of the five-form flux in the bosonic theory (see the considerations about noncommutativity in [7]). One may wonder whether this remark can be confirmed in some independent way. There actually exists a way in the gravity side, although it is very hard to verify it analytically.

The low energy equations of motion for bosonic string theory is

$$
R_{\mu \nu} \sim H_{\mu \lambda \rho} H_{\nu}^{\lambda \rho}
$$

where again we have set the dilaton to 0 , and $H=d B$. Comparing this with (58) we see that the $H^{2}$ term might play the role of the five-form term there. There is however an obstacle. Our VSFT solutions contain a constant B-field and if we replace a constant $B$ in (59) the $H^{2}$ term vanishes. Nevertheless there exists another interpretation. Let us consider for definiteness the vacuum solution, where the droplet is a disk of finite radius $r_{0}$ in the phase space. As we remarked above we can easily reproduce this solution on the VSFT side with a constant field $B=B_{0}$, by taking the limit $N \rightarrow \infty$ such that $\theta_{0} N \sim r_{0}$. Now, the region $r>r_{0}$ corresponds to a vanishing distribution in the large $N$ limit. This can be reproduced as well by taking first $\theta \rightarrow 0$ and then $N \rightarrow \infty$. In other words, in order to reproduce the vacuum solution it is not necessary to assume a constant B field everywhere. Unfortunately we do not know how to deal with VSFT in the presence of a varying background $B$ field. But we believe it is reasonable to assume that, anyhow, well inside the droplet the VSFT solution will be described by the solution with $B=B_{0}$, far outside the droplet by a solution with very large $B$ and in the intermediate region by some interpolating solution. If this is correct then our overall VSFT solution will correspond to a non constant $B$ field: after coarse-graining the profile will be such that the $H^{2}$ term in (59) mimics the five-form quadratic term in (58). Of course one should take care also of the other gravity equations of motion: in order to satisfy them all a nontrivial dilaton might be necessary and the solution is anyhow very likely to be singular. One may object that we are here in presence of two different VSFT solutions (with constant and non constant $B$ field) that correspond to the same space profile. This is true, but the profile we have been talking is the same only in the $\alpha^{\prime} \rightarrow 0$ limit. We expect the $\alpha^{\prime}$ corrections to remove the degeneracy between them.

In any case using bosonic string field theory to establish a correspondence with $1 / 2-\mathrm{BPS}$ states of an $\mathcal{N}=4$ YM theory can be taken, at this stage, only as a suggestion. However the elements we have listed above are striking. So let us suppose that our conjecture is correct at least for the class of states and solutions we are interested in. Then it is convenient to view it in the framework of open-closed string duality. One should not forget that VSFT is a version of open string field theory, i.e. its language is the language of open string theory. 
The correspondence we have established above is between $1 / 2-$ BPS states of SYM theory in $4 \mathrm{D}$ and full VSFT solutions. This suggests that the open string field theory we have been considering describes in fact the physics of open strings attached to the stack of D3-branes where the SYM is defined, that is it is the stringy completion of the latter theory. Once again, the appropriate treatment should make use of superstring field theory. But let us suppose that bosonic SFT is enough for the present purpose; then we must conclude that, using tachyon condensation, we have found a way to pass from open SFT solutions to space time geometry (via coarse-graining), which is one of the major problems in open-closed string duality, and this with the additional bonus of the $\alpha^{\prime}$ corrections.

We would like to add two specifications. The first concerns Chan-Paton factors one is expected to introduce in order to represent a $U(N)$ theory, and we have not. This is in fact not necessary, since it was proven in [52] that VSFT contains solutions with all type of $U(N)$ $\mathrm{CP}$ factors without the need to introduce them by hand. The second concerns the string critical dimension, which, in the bosonic case, is $\mathrm{D}=26$, while the physics of SYM theory lives in $\mathrm{D}=4$. However we have seen in section 3 that our VSFT solution spontaneously solve the problem, because we can choose them translationally invariant in $4 \mathrm{D}$ and of finite or zero size in the transverse dimensions, as need be.

In this paper we have only considered Wigner distributions. As pointed out in [2] there are other proposals, for instance the Husimi distribution, which is based on a convolution of the Wigner one. On the other hand there are many other solutions in VSFT, beside the family based on the sliver and the Laguerre polynomials we have considered so far, for instance the family of butterfly projectors. It would be interesting to see whether our correspondence extends to other phase-space distributions and to other VSFT solutions.

Concerning the future problems to be studied an interesting one relates to the possible utilization of the full VSFT to calculate $\alpha^{\prime}$ corrections. It has been suggested that the superstar solution may develop a horizon due to the stringy corrections. Now, a string state like $|W\rangle$ contains the $\alpha^{\prime}$ corrections to its low energy profile. It is therefore natural to ask whether this knowledge can be translated to the supergravity side. Related to this is the problem of counting the microstates in order to evaluate the entropy of the ensemble, 1, 2, 3. To this end one should be able to count the distinct string fields corresponding to a given low energy profile. A problem nested into this is related to gauge equivalence. The states $\left|\Lambda_{n}\right\rangle$ are defined in terms of a vector $\xi_{n}$ (likewise for the ghost part). These infinitely many numbers $\xi_{n}$ are irrelevant in the low energy limit. This fact is understood in the sense that these numbers are likely to represent only gauge degrees of freedom (otherwise also our previous claim of one-to-one correspondence would seem to need a better phrasing). It would be interesting to find a real proof of this.

\section{Acknowledgments}

L.B. would like to thank A.P.Balachandran, D.Karabali, V.P.Nair and A.P.Polychronakos for useful remarks. This research was supported by the Italian MIUR under the program "Teoria dei Campi, Superstringhe e Gravità" and by CAPES-Brasil as far as R.J.S.S. is concerned. The work of CM is partially supported by IISN - Belgium (convention 4.4505.86), by the "Interuniversity Attraction Poles Programme - Belgian Science Policy" and by the 
European Commission programme MRTN-CT-2004-005104, in which he is associated to V.U. Brussel.

\section{References}

[1] D. Berenstein, Large $N$ BPS states and emergent quantum gravity, arXiv:hep-th/0507203.

[2] V. Balasubramanian, J. de Boer, V. Jejjala and J. Simon, The library of Babel: On the origin of gravitational thermodynamics, arXiv:hep-th/0508023.

[3] P. J. Silva, Rational foundation of GR in terms of statistical mechanic in the AdS/CFT framework, arXiv:hep-th/0508081.

[4] P. G. Shepard, Black hole statistics from holography, JHEP 0510 (2005) 072 arXiv:hep-th/0507260.

[5] J. McGreevy, L. Susskind and N. Toumbas, Invasion of the giant gravitons from anti-de Sitter space, JHEP 0006 (2000) 008 arXiv:hep-th/0003075.

[6] A. Hashimoto, S. Hirano and N. Itzhaki, Large branes in AdS and their field theory dual, JHEP 0008 (2000) 051 arXiv:hep-th/0008016.

[7] S. R. Das, A. Jevicki and S. D. Mathur, Giant gravitons, BPS bounds and noncommutativity, Phys. Rev. D 63 (2001) 044001 arXiv:hep-th/0008088.

[8] R. C. Myers and O. Tafjord, Superstars and giant gravitons, JHEP 0111 (2001) 009 arXiv:hep-th/0109127.

[9] S. Corley, A. Jevicki and S. Ramgoolam, Exact correlators of giant gravitons from dual $N=4$ SYM theory, Adv. Theor. Math. Phys. 5 (2002) 809 arXiv:hep-th/0111222.

[10] O. Aharony, Y. E. Antebi, M. Berkooz and R. Fishman, 'Holey sheets': Pfaffians and subdeterminants as D-brane operators in large $N$ gauge theories, JHEP 0212 (2002) 069 arXiv:hep-th/0211152.

[11] D. Berenstein, A toy model for the AdS/CFT correspondence, JHEP 0407 (2004) 018 arXiv:hep-th/0403110.

[12] H. Lin, O. Lunin and J. Maldacena, Bubbling AdS space and 1/2 BPS geometries, JHEP 0410 (2004) 025 arXiv:hep-th/0409174.

[13] A. Buchel, Coarse-graining 1/2 BPS geometries of type IIB supergravity, arXiv:hep-th/0409271.

[14] N. V. Suryanarayana, Half-BPS giants, free fermions and microstates of superstars, arXiv:hep-th/0411145.

[15] V. Balasubramanian, M. Berkooz, A. Naqvi and M. J. Strassler, Giant gravitons in conformal field theory, JHEP 0204 (2002) 034 arXiv:hep-th/0107119. 
[16] G. Mandal, Fermions from half-BPS supergravity, JHEP 0508 (2005) 052 arXiv:hep-th/0502104.

[17] Y. Takayama and A. Tsuchiya, Complex matrix model and fermion phase space for bubbling AdS geometries, JHEP 0510 (2005) 004 arXiv:hep-th/0507070.

[18] A. Donos, A. Jevicki and J. P. Rodrigues, Matrix model maps in AdS/CFT, arXiv:hep-th/0507124.

[19] A. Boyarsky, B. Kulik and O. Ruchayskiy, Classical and quantum branes in $c=1$ string theory and quantum Hall effect, arXiv:hep-th/0312242.

[20] M. M. Caldarelli and P. J. Silva, Giant gravitons in AdS/CFT. I: Matrix model and back reaction, JHEP 0408 (2004) 029 arXiv:hep-th/0406096.

[21] D. Berenstein, A matrix model for a quantum Hall droplet with manifest particle-hole symmetry, Phys. Rev. D 71 (2005) 085001 arXiv:hep-th/0409115.

[22] A. Ghodsi, A. E. Mosaffa, O. Saremi and M. M. Sheikh-Jabbari, LLL vs. LLM: Half $B P S$ sector of $N=4 S Y M$ equals to quantum Hall system, Nucl. Phys. B 729 (2005) 467 arXiv:hep-th/0505129.

[23] A. Dhar, Bosonization of non-relativstic fermions in 2-dimensions and collective field theory, JHEP 0507 (2005) 064. arXiv:hep-th/0505084.

[24] M. Hillery, R. F. O'Connell, M. O. Scully, E. P. Wigner Distribution functions in physics: fundamentals, Phys.Rep. 106 (1984) 121.

[25] M. V. Berry, Semi-classical Mechanics in Phase Space: A Study of Wigner's Function, Phil. Trans. Royal Soc. London. Series A, Mathematical and Physical Sciences, vol. 287 (1977), 237-271.

[26] S. Giombi, M. Kulaxizi, R. Ricci and D. Trancanelli, Half-BPS geometries and thermodynamics of free fermions, arXiv:hep-th/0512101

[27] M. M. Caldarelli, D. Klemm and P. J. Silva, Chronology protection in anti-de Sitter, Class. Quant. Grav. 22 (2005) 3461 arXiv:hep-th/0411203.

[28] G. Milanesi and M. O'Loughlin, Singularities and closed time-like curves in type IIB 1/2 BPS geometries, JHEP 0509 (2005) 008 arXiv:hep-th/0507056.

[29] L. Rastelli, A. Sen and B. Zwiebach, "Vacuum string field theory," arXiv:hep-th/0106010.

[30] A. Sen, Open-closed duality: Lessons from matrix model, arXiv:hep-th/0308068.

[31] D.J.Gross and A.Jevicki, "Operator Formulation of Interacting String Field Theory", Nucl.Phys. B283 (1987) 1.

D.J.Gross and A.Jevicki, Operator Formulation of Interacting String Field Theory, 2, Nucl.Phys. B287 (1987) 225. 
E.Cremmer,A.Schwimmer, C.Thorn, "The vertex function in Witten's formulation of string field theory, Phys.Lett. 179B (1986) 57.

[32] N. Ohta, "Covariant Interacting String Field Theory In The Fock Space Representation," Phys. Rev. D 34 (1986) 3785 [Erratum-ibid. D 35 (1987) 2627].

[33] L. Bonora, C. Maccaferri, D. Mamone and M. Salizzoni, "Topics in string field theory," arXiv:hep-th/0304270.

[34] A.Leclair, M.E.Peskin, C.R.Preitschopf, "String Field Theory on the Conformal Plane. (I) Kinematical Principles", Nucl.Phys. B317 (1989) 411.

[35] L.Rastelli, A.Sen and B.Zwiebach, "Classical solutions in string field theory around the tachyon vacuum", Adv. Theor. Math. Phys. 5 (2002) 393 [hep-th/0102112].

[36] L.Rastelli, A.Sen and B.Zwiebach, Half-strings, Projectors, and Multiple D-branes in Vacuum String Field Theory, JHEP 0111 (2001) 035 [hep-th/0105058].

[37] N.Moeller, Some exact results on the matter star-product in the half-string formalism, JHEP 0201 (2002) 019 [hep-th/0110204].

[38] L. Bonora, C. Maccaferri and P. Prester, "Dressed sliver solutions in vacuum string field theory," JHEP 0401 (2004) 038 arXiv:hep-th/0311198.]

[39] L. Bonora, C. Maccaferri and P. Prester, "The perturbative spectrum of the dressed sliver," Phys. Rev. D in press arXiv:hep-th/0404154.]

[40] M. Schnabl, Analytic solution for tachyon condensation in open string field theory, arXiv:hep-th/0511286.

[41] G. Moore and W. Taylor "The singular geometry of the sliver", JHEP 0201 (2002) 004 [hep-th0111069].

[42] L. Bonora, D. Mamone and M. Salizzoni, B field and squeezed states in Vacuum String Field Theory, Nucl. Phys. B630 (2002) 163 [hep-th/0201060].

[43] L. Bonora, D. Mamone and M. Salizzoni, "Vacuum String Field Theory with B field", JHEP 0204 (2002) 020 [hep-th/0203188].

[44] L. Bonora, D. Mamone and M. Salizzoni, "Vacuum String Field Theory ancestors of the GMS solitons", JHEP 0301 (2003) 013 [hep-th/0207044].

[45] N.Seiberg and E.Witten, String Theory and Noncommutative Geometry, JHEP 9909, (1999) 032 [hep-th/9908142].

[46] E. Witten, Noncommutative Tachyons and String Field Theory, [hep-th/0006071].

[47] M. Schnabl, String field theory at large B-field and noncommutative geometry, JHEP 0011, (2000) 031 [hep-th/0010034]. 
[48] F. Sugino, Witten's Open String Field Theory in Constant B-field Background, JHEP 0003, (2000) 017 [hep-th/9912254]. T. Kawano, T. Takahashi, Open String Field Theory on Noncommutative Space., Prog.Theor.Phys. 104:459-476,2000 [hep-th/9912274]

[49] T.Kawano and T.Takahashi, Open String Field Theory on Noncommutative Space, Prog. Theor. Phys. 104 (2000) 459 [hep-th/9912274].

[50] J. A. Harvey, Komaba lectures on noncommutative solitons and D-branes, [hepth/0102076].

[51] R. Gopakumar, S. Minwalla and A. Strominger, Noncommutative solitons, JHEP 0005 (2000) 020 [hep-th/0003160].

[52] C. Maccaferri, "Chan-Paton factors and higgsing from vacuum string field theory," JHEP 0509 (2005) 022 arXiv:hep-th/0506213.

[53] I.Ya.Aref'eva, D.M.Belov, A.A.Giryavets, A.S.Koshelev, P.B.Medvedev, Noncommutative field theories and (super)string field theories, hep-th/0111208.

[54] K.Ohmori, A review of tachyon condensation in open string field theories, hep-th/0102085.

K. Ohmori, On ghost structure of vacuum superstring field theory, Nucl. Phys. B 648 (2003) 94 arXiv:hep-th/0208009.

$\mathrm{K}$. Ohmori, Comments on solutions of vacuum superstring field theory, JHEP 0204 (2002) 059 arXiv:hep-th/0204138.

[55] K. Furuuchi and K. Okuyama, "Comma vertex and string field algebra," JHEP 0109 (2001) 035 arXiv:hep-th/0107101.

[56] In preparation. 